\title{
A HIGH-FREQUENCY TUNABLE PIEZOELECTRIC MEMS SCANNER FOR FAST ADDRESSING APPLICATIONS
}

\author{
Paul Janin, Ralf Bauer, Paul Griffin, Erling Riis, and Deepak Uttamchandani \\ University of Strathclyde, Glasgow, UK
}

\begin{abstract}
We present a high-frequency, tunable, piezoelectric MEMS resonant scanner producing an optical scan angle of more than $2^{\circ}$ at frequencies above $100 \mathrm{kHz}$, with noncontact frequency tuning capabilities. The device is fabricated using a cost-efficient multiuser silicon-on-insulator (SOI) process. The scanner uses thin-film piezoelectric aluminium nitride actuators to drive out-of-plane rotation of a $200 \mu \mathrm{m}$ diameter mirror plate through mechanical coupling stages. Up to $3.6 \mathrm{kHz}$ frequency tuning is achieved through on-chip thermally actuated non-contact beam tips placed adjacent to the scanner. The scanner is intended for use in small-scale, fast optomechanical applications requiring careful synchronization through frequency tuning.
\end{abstract}

\section{KEYWORDS}

Optical MEMS, scanning mirrors, piezoelectric, frequency tuning, thermal actuators

\section{INTRODUCTION}

MEMS scanners are a long-standing application of microfabrication techniques to opto-mechanical devices. As individual scanners or array of mirrors, they were successfully implemented in many commercial products, specifically for projection and display systems[1]. Continued development efforts have been made in recent years, aiming at scanners with improved performance in respect to projection display application requirements, with a focus on numerical aperture and angular reach.

Typical MEMS scanners for these applications use various actuation methods and are often resonant systems operating at frequencies under $50 \mathrm{kHz}$ [1], [2], [3]. These speeds are well suited for high-definition displays to be observed by human users, on surface sizes ranging from head-up displays to wall-mounted projection screens[4].

Our work aims to develop MEMS scanners with much higher frequencies (upwards of $100 \mathrm{kHz}$ ) for use in small scale optical systems, in particular for integrated quantum optics. In these applications high speed is essential and the size of the control systems used is crucial for miniaturization while the optical targets themselves - usually atomic particles - are already in the micrometer range and do not require high beam displacements[5], [6]. The device presented for this purpose here is a MEMS resonant scanning mirror with the first scanning frequency at 140.6 $\mathrm{kHz}$, and further scanning modes up to $337 \mathrm{kHz}$.

Furthermore, the device presented in this paper was designed with a frequency tuning mechanism intended to extend the range of operational frequencies available for the scanner, and thus enabling easier synchronization when used with other resonant or generally time-dependent systems.
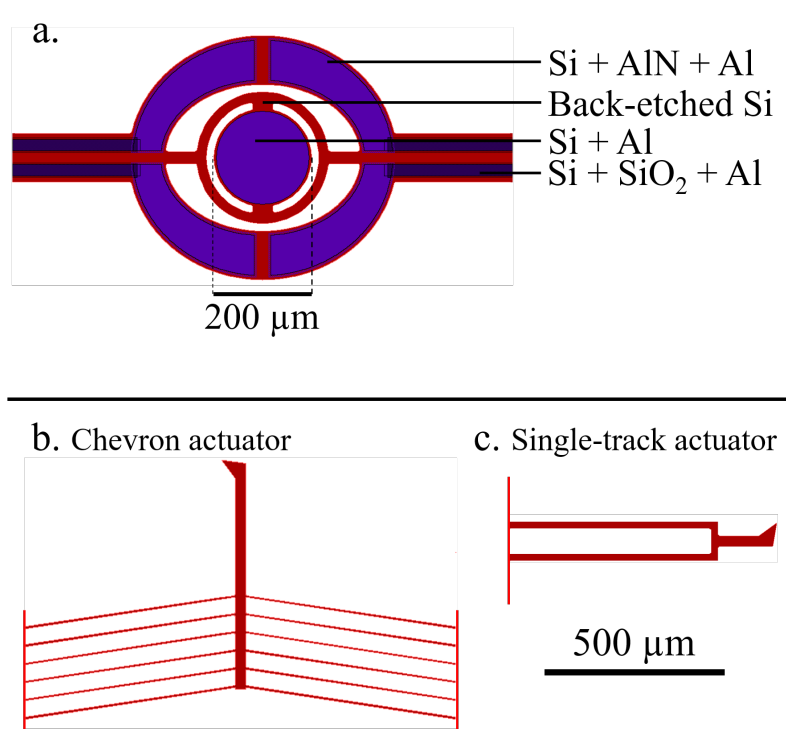

Fig. 1: Top : Schematic layout of the scanning device with the deposited layers highlighted for each area; bottom : layouts of the thermal beam tips, fabricated out of $10 \mu \mathrm{m}$ back etched SOI. Top and bottom not to scale.

We report a tunability range of $3.6 \mathrm{kHz}$, corresponding to a frequency shift of nearly $2.5 \%$ from the original resonance peak, with a moderate reduction in scanning amplitude and an optical scanning range of more than $2^{\circ}$.

\section{DEVICE DESIGN \& FABRICATION}

The presented MEMS device is a piezoelectric scanner based on [7]. The scanner exploits the mechanical coupling between an outer frame holding piezoelectric actuators, a gimbal frame and the mirror plate itself. The mechanical coupling allows to generate an optical scanning range of more than $2^{\circ}$ at frequencies greater than $100 \mathrm{kHz}$, while the use of thin film piezoelectric actuators ensures low power consumption [1]. The scanner mirror surface is $200 \mu \mathrm{m}$ in diameter, while the whole suspended structure is $500 \mu \mathrm{m}$ wide by $1025 \mu \mathrm{m}$ long. Fig. 1a shows a schematic representation of the device. Four aluminium nitride piezoelectric actuator surfaces can be used for actuation, allowing tailored phase and amplitude control of the excitation to gain a maximum angular response.

Independently suspended tuning beams were added around this structure to enable frequency tuning by changing the stiffness of the scanner suspension beams through external control. While the tuning beams have the potential to induce frequency tuning through mechanical contact, the following demonstrates that electrothermal heating and heat conduction effects are the main contributor to the observed frequency changes. 


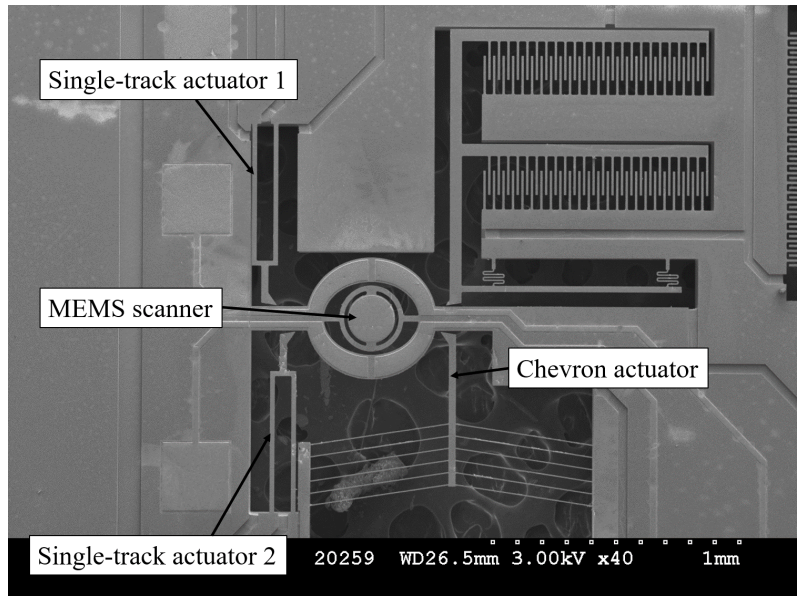

Fig. 2: SEM image of the scanner with the surrounding tuning beams. The comb drive tip (upper right) was not used for tuning in the following.

Two tuning beam tip designs were fabricated: a thermally actuated beam with chevron-shaped suspensions in which the tip displacement is generated by bending from thermal expansion (Fig. 1b), and a single-track, thermally actuated beam intended to push the tip through thermal expansion of the whole tip (Fig. 1c). The beam tips are solely made of $10 \mu \mathrm{m}$ thick single crystalline silicon and were designed to be approximately $10 \mu \mathrm{m}$ away from the scanner suspension beams without actuation. The chevronshaped tuning actuator has an overall length and width of the main tip of $635 \mu \mathrm{m}$ and $30 \mu \mathrm{m}$ respectively. The six chevron connections are each $5 \mu \mathrm{m}$ wide and $590 \mu \mathrm{m}$ long and oriented at a $81^{\circ}$ deg angle to drive a thermal expansion of the actuator towards the scanner suspsion beam. The single-track tuning actuator has a length and width of 20 $\mu \mathrm{m}$ and $740 \mu \mathrm{m}$ respectively, with only the $160 \mu \mathrm{m}$ long tip not undergoing direct Joule heating.

The device was fabricated using the commercial MEMSCAP PiezoMUMPS multi-user fabrication process Piezoelectric actuators are built on a $10 \mu \mathrm{m}$ thick base layer of silicon-on-insulator (SOI), by depositing a thin film of aluminium nitride (AIN) covered by a layer of metallic aluminium to form the top electrode of the actuators. An intermediate layer of silicon oxide is used to insulate the top electrode from the silicon ground plane where piezoelectric material is not needed. The scanning mirror plate is also coated in metallic aluminium for increased reflectivity at visible wavelengths. See Fig. 1a for an illustration of the layer structure of the device. As the final step of fabrication, the substrate is back-etched to form suspended structures capable of out-of-plane displacement without angular limitations. Fig. 2 shows an image of the fabricated device including the multiple tuning actuators placed adjacent to the fast scanner.

\section{FINITE ELEMENT SIMULATION}

We used COMSOL Multiphysics in order to model and simulate the mechanical and thermal response of the device, using finite element method (FEM) simulations. The device geometry and material properties [8] were used to calculate the mechanical eigenmodes of the structure with the corresponding mode shapes. The first eigenfrequencies are shown on Fig. 3 along with the illustration of the expected mode shapes. It should be noted that this simulation was performed using only solid mechanics properties, and does not take in account any external damping or piezoelectric actuation.
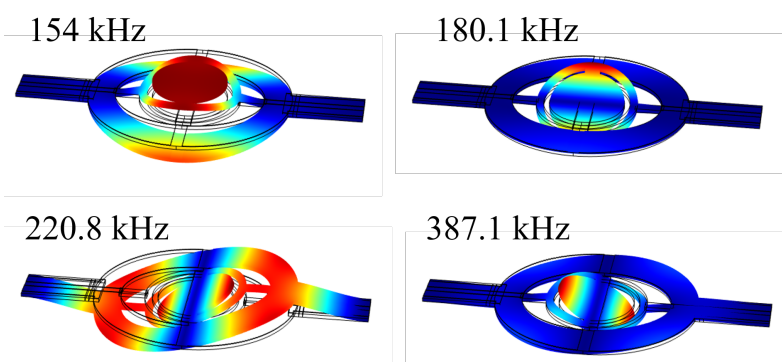

Fig. 3: Simulated mechanical resonance mode shapes of the first four eigenmodes with corresponding eigenfrequencies. Displacements shown are for qualitative illustration and not to scale with device geometry.

\section{THERMAL CHARACTERISATION}

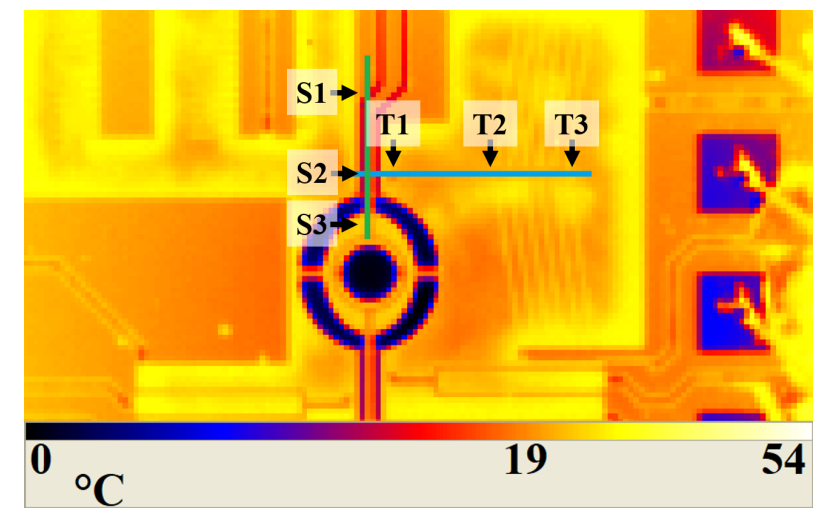

Fig. 4: Thermal image of the device at rest in room temperature. Lines and arrows indicate points used for profile measurements in Fig. 5.

We use a thermal imaging camera (FLIR SC7000 series) to establish the heat distribution within the tuning tips and the device itself. Fig. 4 shows the measurement lines used to establish heat profiles of the device at ambient temperature, while Fig. 5 shows typical profiles obtained when actuating the chevron-shaped tuning beam. Note that due to the limited dynamic range of the camera used and the large difference in temperature between the tip and device structures, their profiles were measured separately. Heat distribution measurements show that the tip comes into contact with the scanner suspension beam with approximately $8.7 \mathrm{~V}$ applied through the chevron tip ; however the small contact area heavily limits the amount of heat transferred via conduction through the material into the device, leading to a large temperature discrepancy. The maximum admissible voltage on the thermal beam tips was found to be approximately $12 \mathrm{~V}$, beyond which the thermal 
suspension beams are subject to thermal breakdown from excessive heat.

The scanner suspension beam profile in Fig. 5 shows a combination of global and localized heating effects on the device. The baseline temperature of approximately $90^{\circ} \mathrm{C}$ measured at the substrate facing side of the the scanner torsion beam, when actuating the chevron thermal tuning actuator with $9.9 \mathrm{~V}$, is consistent with measurements on the chip surface using a thermocouple and is a result of the tip heat spreading through the suspension beams of the thermal actuator and into the silicon bulk. The tip contact point at 22 px exhibits a local temperature increase to $120^{\circ} \mathrm{C}$, carried into the scanner inner suspension beam (from 30px) The temperature dip observed at pixel 7 is an effect of the lower emissivity of the metal track present at this point and does not represent an accurate temperature measurement. Due to the short gap between the tip and the suspension beam - less than $10 \mu \mathrm{m}$ at rest and in the low micrometer or nanometer range during actuation - those localized heating effects persist even without direct contact with the beam tip.

The temperature distribution within the tuning tip itself shows that most of the heat produced by the current circulating through the small suspension beams flows into the central part of the tip (between points $\mathrm{T} 2$ and $\mathrm{T} 3$ on Fig. 5). Temperature quickly drops outside of this area and in particular at the extremity of the tip (point T1). While this distribution is favourable to tip displacement through thermal expansion of the thin beams, it is unfavourable for localized heat transfer as a significant amount is dissipated before reaching the contact point.

\section{OPTO-MECHANICAL CHARACTERISA- TION \& FREQUENCY TUNING RESULTS}

In order to measure the resonance behaviour of the device, we use Doppler vibrometry (Polytec OFV 3000 series), allowing for single-point measurements of velocity while the scanner is being actuated. For sinusoidal movements at a frequency $f$, which is the case for resonant eigenfrequencies, the corresponding displacement amplitude can be calculated as $A=U /(2 \pi f)$ where $U$ is the measured velocity amplitude. In the following, measurements were made at the edge of the mirror plate, at the point of maximum velocity, and the mechanical angular scanning range can be inferred from the relationship $\Theta \approx A /(2 r)$ where $r=100 \mu \mathrm{m}$ is the mirror plate radius, for the typically low angles observed. The mirror is actuated using a single piezoelectric actuator (out of 4) with a $10 \mathrm{~V}_{p p}+5 \mathrm{~V}_{D C}$ sinusoidal voltage applied. The frequency response of the device is shown in Fig. 6. The response was established by measuring the displacement successively at two points of the edge of the central mirror plate, away from the out-of-plane rotation axes. Once the value of the resonant frequencies was known, mode shapes were confirmed by additional measurements on other points of the structure and associated with the mode shapes predicted by simulation.

While the mode shapes and relative displacements of the structure were found to be in good agreement with the simulations, damping effects affected the eigenfrequencies in varying proportions when compared with their predicted
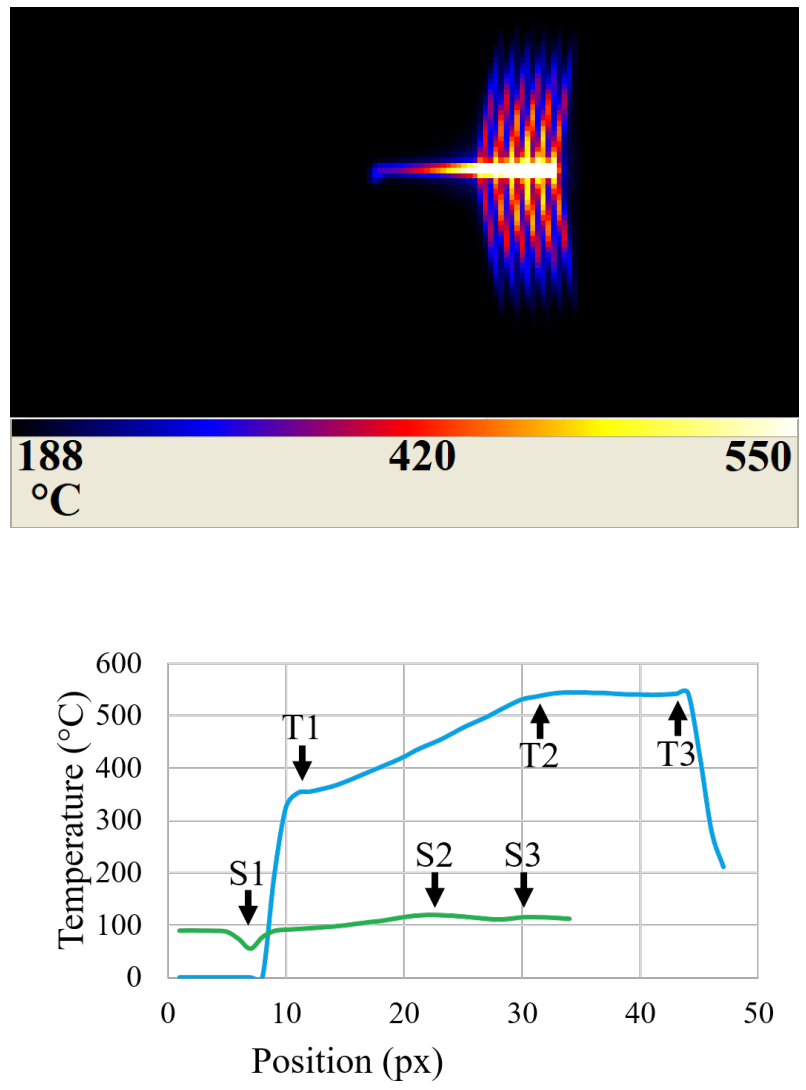

Fig. 5: Top : Thermal image of the device with 8.8 Vdc applied on the thin suspension beams. Note the nonlinear color scale due to the limited camera range. Bottom. measured temperature profile along the lines on Fig. 4. See text for details.

values. Modes involving larger displacements of the gimbal structure saw large reductions in their frequency; the first tipping mode, originally expected at $220.8 \mathrm{kHz}$, saw a $36 \%$ decrease in frequency, while the first tilting mode expected at $180.1 \mathrm{kHz}$ matched its predicted value within a $1 \%$ margin.

Fig. 7 shows the change of a given eigenfrequency with the voltage applied to the chevron type tuning actuator. These measurements were made with $5 \mathrm{~V}_{p p}+2.5 \quad \mathrm{~V}_{d c}$ applied to the piezoelectric actuator, in an effort to limit the hysteresis effects observed for large displacement amplitudes. The eigenfrequency exhibits a roughly quadratic dependency on the tip voltage, consistent with a temperature change through Joule heating along the tip. The step visible around $8.7 \mathrm{~V}$ for the single actuator tuning indicates contact of the tuning tip with the MEMS suspension beam and a subsequent increase in local heat transfer. Furthermore, the use of multiple tips increases the frequency shift, while consistently preserving the quadratic behavior. Further measurements also show that a tip closer to the MEMS frame produces a larger tuning effect, producing the difference shown in Fig. 7.

The measured frequency shift reaches $3 \mathrm{kHz}$ actuating two thermal tips with $8 \mathrm{~V}$, corresponding to approximately 6 times the resonance width for the examined mode before contact is made between the tip and the MEMS structure. 


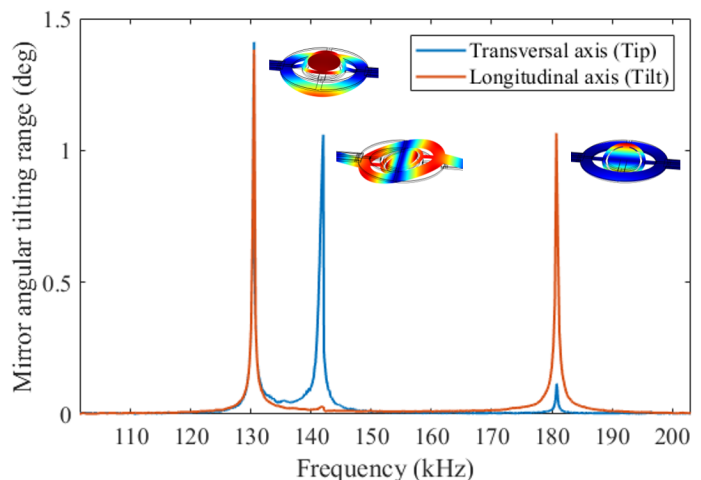

Fig. 6: Frequency response of the scanner in the absence of external influence, as the angular scanning range along each of the out-of-plane rotation axes. The corresponding mode shapes are drawn next to each resonance peak.

This corresponds to an effective frequency range of $3.6 \mathrm{kHz}$, allowing for precise synchronization with other resonant devices ; for instance, with a slower resonant scanner for $2 \mathrm{D}$ raster scanning. It can also be used to correct frequency discrepancies observed among instances of the same device, arising due to fabrication tolerances and variable internal stresses.

The evolution of the frequency response in Fig. 7 also shows a slight decrease in displacement amplitude of about $7 \%$ at maximum voltage for two tips. While this corresponds to a final optical scanning range of $1.02^{\circ}$ (twice the mechanical range), preliminary work shows that voltages much larger than the ones used here can be applied without damage to the device, allowing to keep the scanning range above $2^{\circ}$

\section{ACKNOWLEDGMENTS}

The research presented in this paper was funded by the National Physical Laboratory, and the authors additionally acknowledge funding from the UK Royal Academy of Engineering and EPSRC.

\section{REFERENCES}

[1] S. T. Holmstrom, U. Baran, H. Urey, "MEMS laser scanners: a review", J. of Microelectromech. Syst., vol. 23, no. 2, pp. 259-275, 2014

[2] C. Tsai, Z. Li, Y. Lin and M. S. -. Lu, "A Closed-Loop Controlled CMOS MEMS Biaxial Scanning Mirror for Projection Displays," IEEE Sensors J., vol. 20, no. 1, pp. 242-249, 2020

[3] D. Wang, S. Strassle Rojas, A. Shuping, Z. Tasneem, S. Koppal, S., H. Xie, An "Integrated Forward-View 2-Axis Mems Scanner for Compact 3D Lidar"(2018), in 2018 IEEE 13th Annual International Conference on Nano/Micro Engineered and Molecular Systems (NEMS), Singapore, April 22-26, 2018, pp. 185-188.

[4] Y. H. Seo, K. Hwang, H. Kim, K. H. Jeong, "Scanning MEMS Mirror for high definition and high frame rate Lissajous patterns" Micromachines, vol. 10, no. 1, p. 67,2019
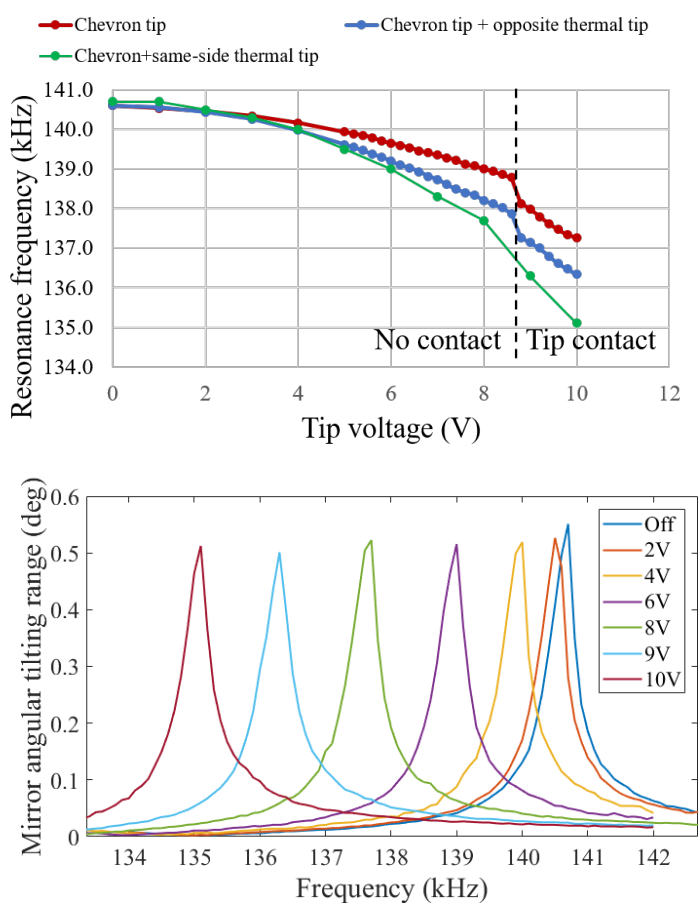

Fig. 7: Top : Evolution of resonance frequency for the first tipping mode. The step at $8.8 \mathrm{~V}$ indicates contact of the chevron tip with the scanner. Bottom : detailed frequency response for varying voltages applied to the chevron tip and the same-side single-track tip. Note the influence of tip position on the frequency drift.

[5] C. Knoernschild, C. Kim, F.P. Lu, J. Kim, ”Multiplexed broadband beam steering system utilizing high speed MEMS mirrors", Optics Express, vol. 17, no. 9, pp. 7233-7244, 2009.

[6] S. Crain, E. Mount, S. Baek, J. Kim, "Individual addressing of trapped $171 \mathrm{Yb}+$ ion qubits using a microelectromechanical systems-based beam steering system", Applied Physics Letters, vol. 105, no. 18, pp. $1-4,2014$

[7] U. Baran, D. Brown, S. T. Holmstrom, D. Balma, W.O. Davis, P. Muralt, H. Urey, "Resonant PZT MEMS scanner for high-resolution displays", $J$. of Microelectromech. Syst., vol. 21, no. 6, pp. 1303-1310, 2012

[8] L. Li, MEMS Micromirrors For Imaging Applications, University of Strathclyde, 2013 